\title{
Parameterization invariance and shape equations of elastic axisymmetric vesicles
}

\author{
R. Podgornik 拥 S. Svetina ${ }^{\ddagger}$ and B. Žekš ${ }^{\ddagger}$ \\ $\dagger$ Laboratory of Structural Biology, \\ Division of Computer Research and Technology, \\ National Institutes of Health, Bethesda, MD 20892 \\ $\ddagger$ Institute of Biophysics, Medical Faculty and J.Stefan Institute, \\ University of Ljubljana, Lipičeva 2, 61105 Ljubljana, Slovenia.
}

\begin{abstract}
The issue of different parameterizations of the axisymmetric vesicle shape addressed by Hu Jian-Guo and Ou-Yang Zhong-Can [ Phys.Rev. E 47 (1993) 461 ] is reassesed, especially as it transpires through the corresponding Euler - Lagrange equations of the associated elastic energy functional. It is argued that for regular, smooth contours of vesicles with spherical topology, different parameterizations of the surface are equivalent and that the corresponding Euler - Lagrange equations are in essence the same. If, however, one allows for discontinuous (higher) derivatives of the contour line at the pole, the differently parameterized Euler - Lagrange equations cease to be equivalent and describe different physical problems. It nevertheless appears to be true that the elastic energy corresponding to smooth contours remains a global minimum.
\end{abstract}

* On leave from: Department of Theoretical Physics, J.Stefan Institute, P.O.Box 100, 61111 Ljubljana, Slovenia. 
Recently $\mathrm{Hu}$ Jian-Guo and Ou-Yang Zhong-Can [四] argued to the effect that different parameterizations of the axisymmetric shape of a lipid vesicle lead to different equations governing its shape. Should this assertion be regarded as generally valid, it leads to disturbing ambiguities in the results of the computation of equilibrium shapes of the elastic vesicles. Also many results derived till now [2, 3] and based on the assumption that the parameterization of the surface does not alter its equilibrium form, would be cast in grave doubt. It is thus of utmost importance to establish the precise limits of the claims made in Ref.1, particularly since it is quite clear from the general theory of the variational calculus [4] that these claims can not be generally valid.

A criticism of the variational methods employed in [1] has already been voiced by Jülicher and Seifert [5], that based their arguments on the incorrect way the boundary terms were treated in the derivation of the variational equations for general topologies. Limiting ourselves to the spherical topology we will, in what follows, investigate the variational shape equations for different parameterizations and their range of validity.

Though all our formal derivations and associated discussion will be centered on the bilayer - couple model, the modifications brought to the formalism in the framework of the spontaneous curvature model - the two are just different limiting cases of the generalized bilayer - couple model - are known [6] and do not in any respect modify the conclusions reached below.

The formulation of the problem treated here is simple. We start with the variational principle for an elastic vesicle under the constraint of constant volume, $V$, constant surface area, $A$, and constant difference between the areas of the two membrane surfaces in contact, $\Delta A$. The equilibrium shape of the vesicle is obtained from the first variation [3]

$$
\delta \mathcal{L}=\delta\left(\mathcal{F}_{b}-\frac{1}{4} L A-\frac{1}{6} M V-\frac{1}{2} N \Delta A\right)=0,
$$

where the bending energy can be expanded in a series with respect to the principal curvatures $c_{1}$ and $c_{2}$ of which the first term is of the form

$$
\mathcal{F}_{b}=\frac{1}{4} \oint\left(c_{1}+c_{2}\right)^{2} d \alpha
$$

In the above equation we omitted the Gaussian elastic term, as we consider only vesicles of fixed (spherical) topology. Furthermore the elastic energy, 
area, volume and area difference are all normalized with respect to the corresponding values for the spherical shape [3], while $L, M$ and $N$ are the Lagrange multipliers corresponding to the constraints of fixed area, volume and area difference. Furthermore $d \alpha$ is the normalized area element of an axisymmetric vesicle, i.e. $\int d \alpha=1$.

We start by choosing the relevant variables describing the shape of an axisymmetric vesicle. These are the angle $\psi(x)$ between the tangent to the contour and the $\mathrm{x}$ - axis, and the contour line itself $z(x)$. The $z$ - axis coincides with the rotational axis and is perpendicular to the $x$ - axis. The connection between the contour line and $\psi(x)$ is

$$
\frac{d z(x)}{d x}=-\tan \psi(x)
$$

while the two principal curvatures of an axisymmetric shape have the form

$$
c_{p}(x)=\frac{\sin \psi(x)}{x} \text { and } c_{m}(x)=\cos \psi(x) \frac{d \psi(x)}{d x} .
$$

The volume and the surface area difference can be obtained in the form

$$
\begin{aligned}
V & =\frac{3}{2} \oint x^{2} c_{p} d \alpha \\
\Delta A & =\frac{1}{2} \oint\left(c_{p}+c_{m}\right) d \alpha .
\end{aligned}
$$

We now analyse four different derivations of the variational shape equations, all stemming from Eq.1, of which three are based on axial radius, area and contour arclength parameterizations, while the last one is a "general" shape equation [7], that for an axisymmetric shape reduces to $\frac{1}{c_{p}}$ parameterization. To the analysis of [1] that contains the comparison of the first and the third of these parameterizations and the "general" shape equation we thus add an additional derivation of the shape equation, where the area itself is taken as a variational parameter.

If we start by taking the axial radius $x$ as the parameter of the shape, we obtain the contour line $z(x)$ from the minimization of the action functional $\mathcal{L}$ which can be cast into a dimensionless form

$$
\mathcal{L}=\frac{1}{8} \int L_{x}\left(x, \psi(x), \frac{d \psi(x)}{d x}\right) d x,
$$


where

$$
\begin{aligned}
L_{x}\left(x, \psi(x), \frac{d \psi(x)}{d x}\right) & =\frac{x}{\cos \psi}\left(\frac{\sin \psi}{x}+\cos \psi \frac{d \psi}{d x}\right)^{2}- \\
& -\frac{1}{\cos \psi}\left(L x+M x^{2} \sin \psi+N x\left(\frac{\sin \psi}{x}+\cos \psi \frac{d \psi}{d x}\right)\right) .
\end{aligned}
$$

The Euler - Lagrange equation corresponding to the $\mathrm{x}$ - parameterization can be obtained from Eq.6 in the form:

$$
\begin{aligned}
\mathcal{H}(x) & \equiv \cos ^{3} \psi(x) \frac{d^{2} \psi(x)}{d x^{2}}-\frac{1}{2} \sin \psi(x) \cos ^{2} \psi(x)\left(\frac{d \psi(x)}{d x}\right)^{2}+\frac{\cos ^{3} \psi(x)}{x}\left(\frac{d \psi(x)}{d x}\right)- \\
& -\frac{x}{2}\left[2 \frac{\sin \psi(x)}{x^{3}}-\left(\frac{\sin \psi(x)}{x}\right)^{3}-M-L \frac{\sin \psi(x)}{x}-N\left(\frac{\sin \psi(x)}{x}\right)^{2}\right]=0 .
\end{aligned}
$$

One notes here that this equation is of the second order with respect to the derivatives of $\psi(x)$. The contour line follows after a solution of Eq. 8 is inserted into Eq.3.

We now proceed with the surface area parameterization of the vesicle shape. We take the axisymmetric area element in the form

$$
d \alpha=\frac{1}{2} \frac{x d x}{\cos \psi(x)},
$$

together with $\psi=\psi(\alpha)$. In this parameterization the Lagrangian is obtained as

$$
\mathcal{L}=\frac{1}{4} \int L_{\alpha}\left(x, \psi(\alpha), \frac{d \psi(\alpha)}{d \alpha}\right) d \alpha
$$

with

$$
\begin{aligned}
L_{\alpha}\left(x, \psi(\alpha), \frac{d \psi(\alpha)}{d \alpha}\right) & =\left(\frac{x}{2} \frac{d \psi}{d \alpha}+\frac{\sin \psi}{x}\right)^{2}-L-M x \sin \psi-N\left(\frac{x}{2} \frac{d \psi}{d \alpha}+\frac{\sin \psi}{x}\right)+ \\
& +\gamma_{\alpha}\left(\frac{x}{2} \frac{d x}{d \alpha}-\cos \psi\right) .
\end{aligned}
$$

The last term in the above equation is due to the constraint Eq.9 in the form of a differential relation between the surface area element and the axial radius. 
In the $\alpha$-parameterization we now obtain two Euler - Lagrange equations, one for $\psi$ field, which is of the second order in the derivatives of $\psi$, and the other one for $\gamma_{\alpha}$ field, which is of the first order. Combining these two equations in analogous way as in Ref.1 into a single equivalent equation for the $\psi$ field of a higher (third) order, we are led to

$$
\frac{d \mathcal{H}(x)}{d x}=0 \rightarrow \mathcal{H}(x)=\text { const. }
$$

which is now the Euler - Lagrange equation in the $\alpha$-parameterization. The $\mathcal{H}(x)$ function is the same as has been defined in Eq.8.

Taking the arclength of the vesicle contour as a parameter, thus

$$
d s=\frac{d x}{\cos \psi(x)}
$$

and $\psi=\psi(s)$, we obtain the Lagrangian in the form

$$
\mathcal{L}=\frac{1}{8} \int L_{s}\left(x, \psi(s), \frac{d \psi(s)}{d s}\right) d s
$$

where

$$
\begin{aligned}
L_{s}\left(x, \psi(s), \frac{d \psi(s)}{d s}\right) & =x\left(\frac{d \psi}{d s}+\frac{\sin \psi}{x}\right)^{2}-L x-M x^{2} \sin \psi-N x\left(\frac{d \psi}{d s}+\frac{\sin \psi}{x}\right)+ \\
& +\gamma_{s}\left(\frac{d x}{d s}-\cos \psi\right)
\end{aligned}
$$

The last term in the above equation again ensures that the geometric constraint Eq.13 is obeyed along the whole of the contour.

The Euler - Lagrange equations are once again two, one for the $\psi$ field and the other one for the $\gamma_{s}$ field. Combining the two of them into a single higher order equation for $\psi$ we derive

$$
\frac{d \mathcal{H}(x)}{d x}+\frac{\mathcal{H}(x)}{x}\left(1-\frac{c_{m}(x)}{c_{p}(x)}\right)=0 \rightarrow \frac{\mathcal{H}(x)}{c_{p}(x)}=\text { const. }
$$

where again $c_{p}$ and $c_{m}$ are the two principal curvatures and the function $\mathcal{H}(x)$ has again been defined in Eq.8. 
The next alternative derivation of the Euler - Lagrange equations for the shape of the vesicle proceeds by describing the variation of the contour line in terms of infinitesimal deformations of the shape in the direction of the local normal to the unperturbed shape [7]. For an axisymmetric shape this is equivalent to taking $\frac{1}{c_{p}}$ as a parameter describing the shape. This procedure leads yet to a new Euler - Lagrange equation, that in the case of an axisymmetric vesicle reduces to Eq.(7) of Ref.1. After some algebra we were able to write the corresponding Euler - Lagrange equation in terms of $\mathcal{H}(x)$, that in this case assumes the form

$$
\frac{d \mathcal{H}(x)}{d x}+\frac{\mathcal{H}(x)}{x}=0 \rightarrow \mathcal{H}(x) x=\text { const }
$$

The same equation has been recently derived by a somewhat different procedure also by Zheng and Liu [8] in their analysis of the axisymmetric shape equations.

We were thus able to show that for these four different derivations of the Euler - Lagrange equations, Eqs.8,12,16 and 17, giving the contour line of the axisymmetric shape, invariably reduce to a general equation of the form $\mathcal{H}(x) f(x)=$ const., where the form of the function $f(x)$ and the value of the constant depend on the type of derivation.

Let us now investigate how the solutions of the different Euler - Lagrange equations differ between themselves. This is most easily seen if we investigate the nature of the contour line close to the pole, i.e. $x \rightarrow 0, s \rightarrow 0, \alpha \rightarrow 0$ in different parameterizations. By expanding the different Euler - Lagrange equations in the vicinity of the pole we get the following limiting behavior. In the $x$ - parameterization the expansion of Eq.8 leads to

$$
c_{p}(x) \cong a+b x^{2}+\ldots,
$$

where the two constants are determined from the constancy of volume $(V)$, surface area $(A)$ and the surface area difference $(\Delta A$ in the bilayer - couple model). In the area parameterization the corresponding limiting behavior following from Eq.12 is

$$
c_{p}(x) \cong a+b|x|+\ldots,
$$

where the constant $a$ is determined from the values of $V, A, \Delta A$, while $b=$ const./3 where the constant is the same as in Eq.12. The arclength param- 
eterization leads to the following limiting law

$$
c_{p}(x) \cong a+b|x|+\ldots,
$$

with $\frac{3 b}{a}=$ const., where the constant is the same as in Eq. 16.

Finally for the Euler - Lagrange equation Eq.17, corresponding to locally normal shape variations introduced by Ou-Yang and Helfrich [7], one gets

$$
c_{p}(x) \cong a \log |x|+\ldots,
$$

with $a=$ const./2, where the constant is the same as in Eq.17. Interestingly enough an equation of the form Eq. 21 has been derived recently by Naito et al. [9] as a general solution (not just a limiting form as is the case in Eq. 21) of their shape equation, which is just the Euler - Lagrange equation in the Ou-Yang - Helfrich parameterization.

One can easily demonstrate that the lowest power $n$ in the expansion $c_{p}(x) \cong a+b x^{n}+\ldots$ compatible with at least one of the four Euler - Lagrange equations is $n=" 0 "$ (in the sense that " $x^{n=0 "}=\log x$ ), corresponding to the Ou-Yang - Helfrich parameterization. On the other hand if we choose the constants in Eqs.12, 16 and 17 as zero, all the $c_{p}$ 's reduce to the limiting form Eq. 18.

The contour line close to the poles is then obtained in an approximate form as

$$
\frac{d z(x)}{d x}=-\tan \psi(x) \cong-\left(x c_{p}(x)\right) .
$$

It is thus clear that only the form Eq.18 keeps the contour line and all its higher derivatives finite close to the pole. The other limiting forms lead to discontinuities in the third derivative of the form

$$
\frac{d^{3} z(x)}{d x^{3}} \cong-2 b \operatorname{sgn}(x)
$$

for the limiting laws Eqs. 19 and 20, or of the form

$$
\frac{d^{3} z(x)}{d x^{3}} \cong-\frac{a}{|x|} \operatorname{sgn}(x)
$$

for the limiting case Eq.21.

The existence of discontinuities in (higher) derivatives of the contour $z(x)$ is in general connected with point source terms in the Lagrangian. Let us 
investigate this connection in the case of the Ou-Yang - Helfrich parameterization. An external force would in general introduce a term of the form

$$
\mathcal{F}=\mathcal{F}_{b}+\frac{1}{4} \int f(x) z(x) d x
$$

into the energy of the vesicle, where $f(x)$ is the linear density of the force in the direction of $\mathrm{z}$ - axis and the numeric coefficient has been chosen for later convenience (if there were external momenta acting on the contour we would in general have to include those contributions too). As Eq. 24 refers to a discontinuity at the pole, we presume that the force density has the form $f(x)=f_{0} \delta(x)$, where $f_{0}$ is a constant. Deriving now the Euler - Lagrange equation for the ansatz Eq.25 we can cast it in the familiar form

$$
\mathcal{H}(x) x=f_{0} .
$$

The constant in Eq.17 is thus nothing but the magnitude of the point external force acting at both poles. Instead of using the language of point sources in the Lagrangian we could also invoke external constraints: if the separation between the poles is kept constant $\Delta z=$ const., then $f_{0}$ is just the conjugate variable to $\Delta z$.

Either way the conclusion is that the non-analyticities of the $\mathrm{Hu}$ - $\mathrm{Ou}$ Yang type in the derivatives of the contour line at the poles of the form Eq.24 exist only if there are external forces acting on the poles, pulling (pushing) them apart (together). More complicated external sources (constraints) are needed in the case of other parameterizations.

Another conclusion pertinent to the above discussion is that no matter what the magnitude of $f_{0}$ might be, the corresponding membrane bending energy will always be larger than in the $f_{0}=0$ case. On Fig. 1 we present a result of numerical computation of $\mathcal{F}_{b}$ Eq.2 for a range of $f_{0}$ values while keeping $V$ and $\Delta A$ constant ( $V=0.95$ and $\Delta A=1.0129$ ). Obviously $f_{0}=0$ case represents a global minimum of this energy.

One is thus lead to the following conclusion regarding the different forms of the Euler - Lagrange equation, stemming from the various parameterizations of the vesicle shape. If one demands that the contour line be a smooth function, without any discontinuities in its values or its derivatives, then all the different Euler - Lagrange equations reduce to a single equation of the form $\mathcal{H}(x)=0$, identical to the $\mathrm{x}$ - parameterization result. This is consistent with the parameterization invariance of the variational problem, but 
is at odds with the generality of the claims made in Ref.1. On the other hand if one allows for discontinuities in the (higher) derivatives of the contour line of the vesicle, then each Euler - Lagrange equation corresponding to different parameterizations gives different equilibrium shapes. However, these shapes correspond to different physical problems. It appears that only the parameterization where $\mathcal{H}(x)=0$ describes a completely unconstrained vesicle of spherical topology. The other parameterizations describe the shape of a vesicle under external forces and are not relevant to the problem of the shapes of an unconstrained vesicle, but might provide additional insight into the shapes of constrained vesicles.

The conclusion of this paper, contrary to the assesments of [1], is that for "smooth" axisymmetric shapes the way in which one derives the shape equations matters little. All the different parameterizations employed in the literature are equally correct and their corresponding variational equations are parameterization invariant - a results of the general theory of variational calculus of long standing [4]. Should one, however, want to include 'pathological' shapes with various discontinuities in the higher derivatives of the contour line one should first of all start with a more elaborate form of the curvature energy including the external forces or geometrical constraints that lead in each particular case to these discontinuities. For these cases no general form of the free energy is, however, presently in sight and the discussion of the variational equations for those cases should reflect the explicit constraints for each particular case.

\section{References}

[1] Hu Jian-Guo, Ou-Yang Zhong-Can, Phys. Rev. E 47 (1993) 461

[2] H.J. Deuling, W. Helfrich, J.Phys. (Paris) 37 (1076) 1335

[3] S.Svetina, B.Žekš, Eur. Biophys. J. 17 (1989) 101

[4] A.R.Forsyth, Calculus of Variations (Dover, New York, 1960)

[5] F. Jülicher, U. Seifert, Phys.Rev. E 49 (1994) 4728

[6] U.Seifert, K.Berndl, R.Lipowsky, Phys. Rev. A 44 (1991) 1182 
[7] Ou-Yang Zhong-Can, W.Helfrich, Phys. Rev. A 39 (1989) 5280

[8] Wei-Mou Zheng, Jixing Liu, Phys. Rev. E 48 (1993) 2856

[9] Hiroyoshi Naito, Masahiro Okuda, Ou-Yang Zhong-can, Phys. Rev. E 48 (1993) 2304

\section{Figure Captions}

Fig.1: Membrane bending energy $\mathcal{F}_{b}$ obtained from numerical integration of Eq.26 for different values of the force $f_{0}$. The normalized volume is $V=0.95$ and the normalized area difference is $\Delta A=1.0129$.

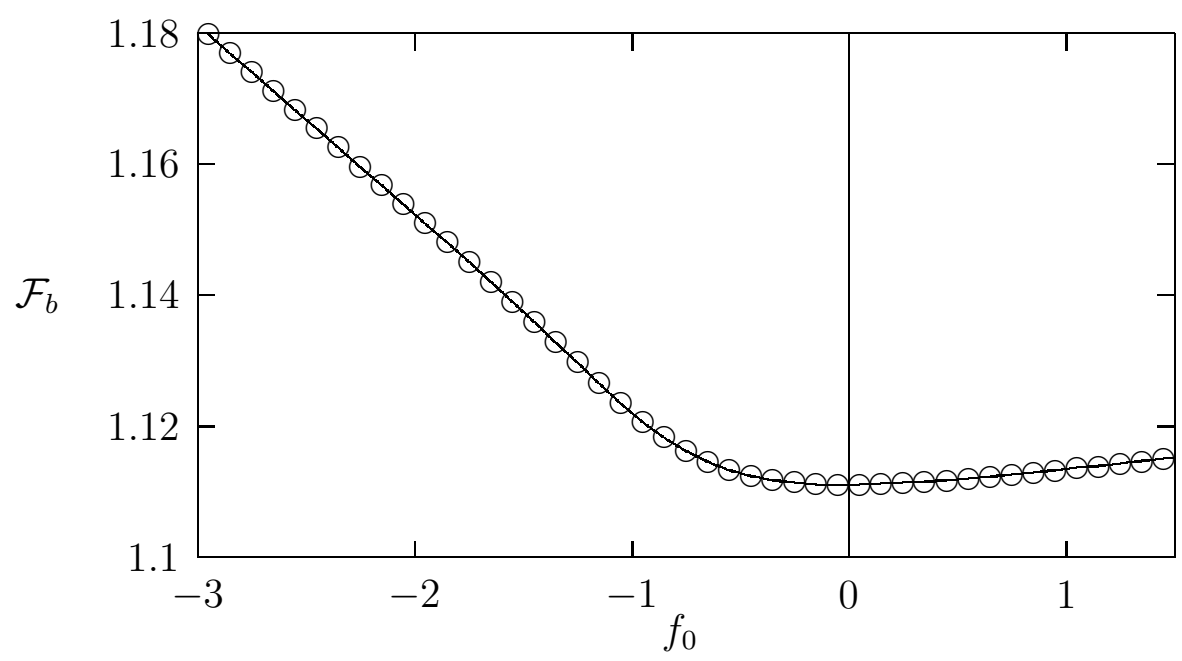

